Pacific Journal of Mathematic 


\title{
A NOTE ON THE AUTOMORPHISM GROUPS OF SIMPLE DIMENSION GROUPS
}

\author{
CHAO-LiANG SHEN
}

\begin{abstract}
We study the automorphism of simple dimension groups with underlying group $\boldsymbol{Z}^{2}$ (which are of the form $\left(Z^{2}, P_{\alpha}\right), \alpha$ a positive irrational) and find out the necessary and sufficient condition for the dimension group $\left(Z^{2}, P_{\alpha}\right)$ having nontrivial automorphisms is $\alpha$ being a quadratic surd. In this case we also obtain the classification of the automorphism via the Bratteli diagram of the dimension groups.
\end{abstract}

Introduction. In his paper [6], George Elliott proposed the notion of dimension groups for the approximately finite $C^{*}$-algebras (AF algebras) which were originally studied by J. Glimm, J. Dixmier, and O. Bratteli [1]. He showed that the classification of the dimension groups is equivalent to the stable classification of $\mathrm{AF}$ algebras. He also found that the dimension groups satisfy the Riesz interpolation property (see §1) and raised the problem of intrinsic characterization of dimension groups. An ordered group (see §1) which satisfies the Riesz interpolation property is called a Riesz group. It is shown by Effros, Handelman, and the author [5] that Riesz groups are in fact dimension groups.

Although we know that Riesz groups are dimension groups, for the practical applications, we still have to have a method for finding the corresponding Bratteli diagram [1]. In [3], [4], we have studied this problem for certain classes of dimension groups. On the other hand, it is also important to understand the structure of the automorphisms of $\mathrm{AF}$ algebras and the relation of those to the automorphisms of the corresponding dimension groups. This question has been studied by Elliott [6], Effros and Rosenberg [2] (also see 15]). By their results, we see that automorphism of dimension groups tells us a certain amount of information about the approximately inner automorphisms of AF algebras.

In this note, we study the automorphism groups of the dimension groups of the form $\left(\boldsymbol{Z}^{2}, P_{\alpha}\right)$ (see $\S 1$ for definition) where $\alpha$ is irrational. In this case, the continued fraction analysis of [3] makes theory particularly transparent. We also provide some information about the automorphisms of higher rank simple dimension groups. In $\$ 2$, we give a necessary and sufficient condition for when the automorphism group is nontrivial, we also obtain the classification of the automorphisms via the Bratteli diagram of the dimension groups. As pointed out in [6], [5], the automorphism groups of 
dimension groups have certain relations to the algebraic units in the given groups. In $\S 3$, we make this correspondence explicit. At the end, we mention some unsolved problems.

Finally, the author wishes to thank his teacher and advisor Professor Edward G. Effros for his encouragement and suggestions.

1. Preliminaries, In this section we shall recall some definitions and theorems which we shall use in $\S \S 2,3$.

By an ordered group we shall mean an abelian group $G$ together with a subset $G^{+}$(called the positive cone of $G$ ) such that $0 \in G^{+}$, $G^{+}+G^{+} \subset G^{+}, G^{+}-G^{+}=G$, and for $n \in N, n g \in G^{+}$implies $g \in G^{+}$. We shall denote $g \geqq 0$ if $g \in G^{+}$. By an order homomorphism $\Psi: G \rightarrow$ $H$, where $G$ and $H$ are ordered groups, we mean a group homomorphism $\Psi$ with the property: $\Psi\left(G^{+}\right) \subset H^{+}$. By an order isomorphism $\Psi: G \rightarrow H$ we mean that $\Psi$ is a group isomorphism and $\Psi\left(G^{+}\right)=H^{+}$. $\Psi$ is an (order) automorphism of $G$ if $\Psi: G \rightarrow G$ is an order isomorphism. The group of automorphisms of $G$ shall be denhted by AUH $G$.

Given the group $Z^{n}$, we assign it an ordering, which is defined by the positive cone

$$
\left(\boldsymbol{Z}^{n}\right)^{+}=\left\{\left(a_{1}, \cdots, a_{n}\right): a_{i} \geqq 0 \text { for all } i\right\} ;
$$

the group $\boldsymbol{Z}^{n}$ together with the above ordering is called a simplicial group. Given an inductive sequence $\left\{\boldsymbol{Z}^{n}, \psi_{k_{n} k+1}^{k+1}\right\}$, where $\boldsymbol{Z}^{n_{k}}$ are simplicial groups and $\varphi_{k, k+1}: \boldsymbol{Z}^{n_{k}} \rightarrow \boldsymbol{Z}$ are order homomorphisms, we define an ordering on $\lim _{\rightarrow}\left\{\boldsymbol{Z}^{n_{k}}, \Psi_{k, k+1}\right\}=G$ by letting $G^{+}=\bigcup_{k \geqq 1} \theta_{k}\left(\left(\boldsymbol{Z}^{n_{k}}\right)^{+}\right)$, where $\theta_{k}: Z^{n_{k}} \rightarrow G$ are the canonical homomorphisms. The group $G$ together with the ordering assigned above is call a dimension group.

A dimension group $G$ must satisfy the following Riesz interpolation property: If $a, b \leqq c, d$ in $G$, then there exists an $e$ in $G$ such that $a, b \leqq e \leqq c, d$. A dimension group $G$ is simple if for any $a \in$ $G^{+} \backslash\{0\},\{b \in G: 0 \leqq b \leqq n a$ for some $n \in N\}=G^{+}$. We have the following

Theorem 1.1. If $\left(\boldsymbol{Z}^{2}, P\right)$ is a simple dimension group, then it must be totally ordered. Furthermore, one may assume that the positive cone $P$ is given by

$$
P=\{(x, y): \alpha x+y \geqq 0\},
$$

where $\alpha$ is a positive irrational number. We denote $P$ by $P_{\alpha} . \quad\left(Z^{2}\right.$, $\left.P_{\alpha}\right)$ is order isomorphic to $\left(\boldsymbol{Z}^{2}, P_{\beta}\right)$ if and only if there exists $\Psi=$ $\left[\begin{array}{ll}a & c \\ b & d\end{array}\right] \in G L(2, Z)$ such that

(1) $\alpha a+b>0, \alpha c+d>0$,

(2) $\beta=(\alpha a+b) /(\alpha c+d)$. 
Furthermore, $\varphi$ is an order automorphism of $\left(\boldsymbol{Z}^{2}, P_{\alpha}\right)$ if and only if

(i) $\phi \in \mathrm{GL}(2, \boldsymbol{Z})$,

(ii) if we write $\rho=\left[\begin{array}{ll}a & c \\ b & d\end{array}\right]$, then $\alpha a+b>0, \alpha c+d>0$, and $\alpha=(\alpha a+b) /(\alpha c+d)$.

Proof. We need only prove the last part of the theorem, for the rest, see [10, Prop. 4.5], [10, Lemma 4.7], [3, Th. 2.1].

If $\varphi$ is an automorphism of $\left(\boldsymbol{Z}^{2}, P_{\alpha}\right)$, it is clear that $\varphi \in \mathrm{GL}(2, \boldsymbol{Z})$. Let us write $\varphi=\left[\begin{array}{ll}a & c \\ b & d\end{array}\right]$. Since $(1,0),(0,1) \in P_{\alpha}$, if $\varphi$ is an order automorphism, we must have $\varphi(1,0), \varphi(0,1) \in P_{\alpha}$, and hence $\alpha a+b>$ $0, \alpha c+d>0$. On the other hand, since $\varphi$ is an order automorphism, $(x, y) \in P_{\alpha}$ if and only if $\varphi(x, y) \in P_{\alpha}$, i.e., $\alpha x+y \geqq 0$ if and only if $\alpha(a x+c y)+(b x+d y) \geqq 0$, i.e., if and only if $(\alpha a+b) x+(\alpha c+d) y \geqq 0$. Since $\alpha c+d>0$, the latter is equivalent to

$$
((\alpha a+b) /(\alpha c+d)) x+y \geqq 0 .
$$

Thus $\alpha=(\alpha a+b) /(\alpha c+d)$. The converse is obvious. (Also see the proof of [10, Lemma 4.7]).

Given an irrational number $\alpha \in \boldsymbol{R}^{+}$, we can associate with it a unique simple continued fraction (see [8])

$$
\left[a_{0}, a_{1}, \cdots\right]
$$

where $a_{0} \in Z^{+}, a_{n} \in N$ for all $n \geqq 1$, such that if we define

$$
\begin{aligned}
p_{0} & =a_{0}, p_{1}=a_{1} a_{0}+1, \cdots, p_{n}=a_{n} p_{n-1}+p_{n-2}, \\
q_{0} & =1, q_{1}=a_{1}, \cdots, q_{n}=a_{n} q_{n-1}+q_{n-2},
\end{aligned}
$$

then $p_{2 n} / q_{2 n}$ is an increasing sequence, $p_{2 m-1} / q_{2 m-1}$ is a decreasing seqtence, $p_{2 n} / q_{2 n}<p_{2 m-1} / q_{2 m-1}$ for all $n, m$, and

$$
\lim p_{2 n} / q_{2 n}=\lim p_{2 m-1} / q_{2 m-1}=\alpha .
$$

We have

THEOREM 1.2 [3, Th. 3.2]. Let $\left[a_{0}, a_{1}, \cdots\right]$ be the simple continued fraction expansion of $\alpha$, and define $\Psi_{k}: Z^{2} \rightarrow Z^{2}$ by the matrix $\left[\begin{array}{ll}a_{k} & 1 \\ 1 & 0\end{array}\right]$. Then $\underset{\rightarrow}{\lim }\left\{\boldsymbol{Z}^{2}, \psi_{k}\right\}=\left(\boldsymbol{Z}^{2}, P_{\alpha}\right)$.

Two irrational numbers $\alpha$ and $\beta$ are equivalent if their simple continued fractions agree after some stage. Theorem 1.1 just means that $\alpha$ and $\beta$ are equivalent.

A simple continued fraction $\left[a_{0}, a_{1}, \cdots\right]$ is periodic if $a_{m}=a_{m+k}$ 
for a fixed $k$ and for all $m \geqq L$, we use the notation $\left[a_{0}, a_{1}, \cdots\right]=$ $\left[a_{0}, a_{1}, \cdots, a_{L-1}, \dot{a}_{L}, \cdots, \dot{a}_{L+k-1}, \cdots\right]$, where $a_{L}, \cdots, a_{L+k-1}$ is a period. Replacing a periodic irrational number $\alpha$ by an equivalent irrational if necessary, we may assume that its period starts from $a_{0}$, i.e., $\alpha=$ $\left[\dot{a}_{0}, \cdots, \dot{a}_{L-1}, \cdots\right]$.

For the periodic irrational numbers, we have the following

THEOREM 1.3 [8, Ths. 176, 177]. $\alpha$ is a periodic irrational number if and only if it is a zero of a Q-irreducible integral quadratic polynomial, which has postive discriminant.

THEOREM 1.4 [8, Th. 172]. $\alpha$ is irrational. If $\left[\begin{array}{ll}a & c \\ b & d\end{array}\right] \in \mathrm{GL}(2, Z)$ such that $c>d>0$ and $\alpha=(\beta a+b) /(\beta c+d)$, and $\left[a_{0}, \cdots, a_{n}\right]$ the simple continued fraction of a/c, then there exist $a_{n+1}, \cdots \in N$ such that

(1) $\alpha=\left[a_{0}, \cdots, a_{n}, a_{n+1}, \cdots\right]$ is the simple continued fraction of $\alpha$, and $\beta=\left[a_{n+1}, \cdots\right]$,

(2) $a=p_{n}, b=p_{n-1}, c=q_{n}, d=q_{n-1}$.

LEMMA $1.5\left[8\right.$, p. 140]. If $\alpha=\left[\dot{\alpha}_{0}, \cdots, \dot{\alpha}_{L-1}, \cdots\right]$, then $\alpha=$ $\left(p_{L-1} \alpha+p_{L-2}\right) /\left(q_{L-1} \alpha+q_{L-2}\right)$.

2. The classification of the automorphisms of $\left(Z^{2}, P_{\alpha}\right)$.

THEOREM 2.1. $\left(\boldsymbol{Z}^{2}, P_{\alpha}\right)$ has a nontrivial automorphism if and only if $\alpha$ is a periodic irrational number.

Proof. By Theorem 1.1, $\psi \in \operatorname{AUT}\left(Z^{2}, P_{\alpha}\right)$ if and only if $\psi=$ $\left[\begin{array}{ll}a & c \\ b & d\end{array}\right] \in \mathrm{GL}(2, Z)$, where $\alpha a+b>0, \alpha c+d>0$, and $\alpha=(\alpha a+b) /(\alpha c+d)$. If $\psi$ is nontrivial, then $c \neq 0$, otherwise $\alpha=\alpha(a / d)+(b / d)$ implies $a=d, b=0$, and $\Psi$ is trivial. Thus we have

$$
c \alpha^{2}+(d-a) \alpha-b=0,
$$

i.e., $\alpha$ is periodic.

Conversely, given $\alpha=\left[\dot{a}_{0}, \cdots, \dot{a}_{L-1}, \cdots\right]$, we have $\alpha=\left[a_{0}, \cdots\right.$, $\left.a_{L-1}, \alpha\right]$, and thus $\alpha=\left(p_{L-1} \alpha+p_{L-2}\right) /\left(q_{L-1} \alpha+q_{L-2}\right)$ by Lemma 1.5. Letting $\Psi_{\alpha}=\left[\begin{array}{ll}p_{L-1} & q_{L-1} \\ p_{L-2} & q_{L-2}\end{array}\right]$, by Theorem 1.1, and $q_{L-1} \neq 0, \Psi_{\alpha}$ is a desired automorphism.

Definition 1. The automorphism $\Psi_{\alpha}$ in Theorem 2.1 is called the canonical automorphism of $\left(Z^{2}, P_{\alpha}\right)$. 
By Theorem 2.1 we see that the continued fraction of provides us a nontrivial automorphism $\Psi_{\alpha}$. We note that the automorphism $\Psi_{\sqrt{2}}$ is precisely the automorphism of $\left(\boldsymbol{Z}^{2}, P_{\sqrt{2}}\right)$ studied by Elloitt in [6]. It is very natural to ask the question whether all automorphisms arise in this manner. We shall give an affirmative answer in Theorem 2.3 .

Lemma 2.2. Given $\alpha=\left[\dot{a}_{0}, \cdots, \dot{a}_{L-1}, \cdots\right]$. Then

$$
\Psi_{\alpha}^{n}=\left[\begin{array}{ll}
p_{n L-1} & q_{n L-1} \\
p_{n L-2} & q_{n L-2}
\end{array}\right], \quad n \geqq 1
$$

Proof. We shall use induction on $n$. If $n=1$ this is trivial. Assume that

$$
\Psi_{\alpha}^{n}=\left[\begin{array}{ll}
p_{n L-1} & q_{n L-1} \\
p_{n L-2} & q_{n L-2}
\end{array}\right], \quad \Psi_{\alpha}^{n+1}=\left[\begin{array}{ll}
p_{L-1} & q_{L-1} \\
p_{L-2} & q_{L-2}
\end{array}\right]\left[\begin{array}{ll}
p_{n L-1} & q_{n L-1} \\
p_{n L-2} & q_{n L-2}
\end{array}\right] .
$$

Observe that (see $[3, \S 3]$ )

$$
\begin{aligned}
{\left[\begin{array}{ll}
p_{(n+1) L-1} & q_{(n+1) L-1} \\
p_{(n+1) L-2} & q_{(n+1) L-2}
\end{array}\right] } & =\left[\begin{array}{cc}
a_{(n+1) L-1} & 1 \\
1 & 0
\end{array}\right]\left[\begin{array}{ll}
p_{(n+1) L-2} & q_{(n+1) L-2} \\
p_{(n+1) L-3} & q_{(n+1) L-3}
\end{array}\right] \\
& =\cdots \\
& =\left[\begin{array}{cc}
a_{(n+1) L-1} & 1 \\
1 & 0
\end{array}\right] \ldots\left[\begin{array}{ll}
a_{n L} & 1 \\
1 & 0
\end{array}\right]\left[\begin{array}{ll}
p_{n L-1} & q_{n L-1} \\
p_{n L-2} & q_{n L-2}
\end{array}\right] \\
& =\left[\begin{array}{cc}
a_{L-1} & 1 \\
1 & 0
\end{array}\right] \ldots\left[\begin{array}{ll}
a_{0} & 1 \\
1 & 0
\end{array}\right]\left[\begin{array}{ll}
p_{n L-1} & q_{n L-1} \\
p_{n L-2} & q_{n L-2}
\end{array}\right] \\
& =\left[\begin{array}{cc}
p_{L-1} & q_{L-1} \\
p_{L-2} & q_{L-2}
\end{array}\right]\left[\begin{array}{ll}
p_{n L-1} & q_{n L-1} \\
p_{n L-2} & q_{n L-2}
\end{array}\right] \\
& =\Psi_{\alpha}^{n+1} .
\end{aligned}
$$

Thus we are done.

THEOREM 2.3. If $\Psi=\left[\begin{array}{ll}a & c \\ b & d\end{array}\right] \in \operatorname{AUT}\left(Z^{2}, P_{\alpha}\right), \alpha$ periodic, then $\Psi=$ $\Psi_{\alpha}^{n}$ for some $n \in Z$.

Proof. $\Psi \in \operatorname{AUT}\left(Z^{2}, P_{\alpha}\right)$, we have $(\alpha a+b) /(\alpha c+d)=\alpha$.

Case 1. $c>d>0$ : In this case, by applying Theorem 1.4 we have $a=p_{n}, b=p_{n-1}, c=q_{n}, d=q_{n-1}$ for some $n \in N$, and thus $n=$ $k L-1$ for some $k \in N$, where $a_{0}, \cdots, a_{L-1}$ form a period of the simple continued fraction of $\alpha$. Thus, by Lemma 2.2, we have 


$$
\Psi=\left[\begin{array}{ll}
p_{k L-1} & q_{k L-1} \\
p_{k L-2} & q_{k L-2}
\end{array}\right]=\Psi_{\alpha}^{k} .
$$

Case 2. $c>0, d>0$ : Let

$$
\Psi^{\prime}=\left[\begin{array}{ll}
p_{L-1} & q_{L-1} \\
p_{L-2} & q_{L-2}
\end{array}\right] \Psi .
$$

Since $p_{L-1} c+q_{L-1} d>p_{L-2} c+q_{L-2} d>0$, the previous Case 1 implies that $\Psi^{\prime}=\Psi_{\alpha}^{k+1}$ for some $k$, and thus $\Psi=\Psi_{\alpha}^{k}$.

Case 3. No restriction on $c, d$ : Choose $k$ sufficiently large such that $p_{k L-1} / q_{k L-1}>1, p_{k L-2} / q_{k L-2}>1$ (this is legitimate since $\alpha>1$ ) and $c p_{k L-i}+d q_{k L-i}>0, i=1,2$ (this is also legitimate since $c \alpha+d>0$, and $\left.\lim \left(p_{k L-i} / q_{k L-i}\right)=\alpha\right)$. Let

$$
\begin{aligned}
\Psi^{\prime \prime}=\Psi_{\alpha}^{k} \Psi & =\left[\begin{array}{ll}
p_{k L-1} & q_{k L-1} \\
p_{k L-2} & q_{k L-2}
\end{array}\right]\left[\begin{array}{ll}
a & c \\
b & d
\end{array}\right] \\
& =\left[\begin{array}{ll}
* & p_{k L-1} c+q_{k L-1} d \\
* & p_{k L-2} c+q_{k L-2} d
\end{array}\right] .
\end{aligned}
$$

$\Psi^{\prime \prime}$ is of the form considered in Case 2. Thus $\Psi^{\prime \prime}=\Psi_{\alpha}^{m}$ for some $m \in \boldsymbol{Z}$. Hence $\Psi=\Psi_{x}^{m-k}$.

For the simple dimension group $\left(Z^{n}, P\right), n \geqq 2$, we have the following result (this was suggested by Edward G. Effros).

TheOREM 2.4. Suppose that $\Psi \in \mathrm{GL}(n, Z)$ has strictly positive entries. Let $G$ be the inductive limit group of the sequence

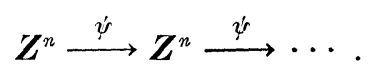

Then AUT $G$ is nontrivial, and $\Psi \in$ AUT $G$.

Proof. Since the connecting isomorphisms are constant, a result of Elliott implies that $G$ is a simple dimension group with a unique extreme state $\eta$ (see [4, Prop. 2.2]) (roughly speaking, this means that $\left.G^{+}=\left\{\left(z_{1}, \cdots, z_{n}\right) \in \boldsymbol{Z}^{n}: \eta\left(z_{1}, \cdots, z_{n}\right)>0\right\} \cup\{(0, \cdots, 0)\}\right)$. We also know that, if we view $\eta$ as a vector in $R^{n}, \eta$ is an eigen vector of the Perron eigenvalue $\lambda$ of $\Psi^{*}$ (see $[4, \S 2]$ ). Given

$$
\begin{aligned}
a \in G^{+} \backslash\{0\}, \eta(\Psi(a)) & =\Psi^{*}(\eta)(a)=\lambda \eta(a)>0, \eta\left(\Psi^{-1}(a)\right) \\
& =\left(\Psi^{-1}\right)^{*}(\eta)(a)=\left(\Psi^{*}\right)^{-1}(\eta)(a)=\lambda^{-1} \eta(a)>0 .
\end{aligned}
$$

Thus $\Psi \in$ AUT $G$. 
REMARK. Another proof can also be derived by using the definition of the positive cone of $G$.

By using [5, $\$ 4$, it is easy to see that if $\left(\boldsymbol{Z}^{n}, P\right)$ is a simple, totally ordered group, then $\operatorname{AUT}\left(\boldsymbol{Z}^{n}, P\right)$ is abelian. Given any simple dimension group $\left(\boldsymbol{Z}^{n}, P\right)$, it is still not clear to us how to determine the generators for $\operatorname{AUT}\left(Z^{n}, P\right)$, when $n>2$. It seems to us that the number of generators would be determined by the Diophantine properties of the extreme states of $\left(\boldsymbol{Z}^{n}, P\right)$ (i.e., it will depend upon whether or not the coefficients of the extreme states are algebraic or transcendental).

3. Algebraic units in a real quadratic field and the automorphism group of $\left(\boldsymbol{Z}^{2}, P_{\alpha}\right)$. An alternative approach to the study of $\operatorname{AUT}\left(Z^{n}, P\right)$ is provided by the theory of algebraic units (see [5]). Since various difficulties arise in the continued fraction theory for $n>2$ (see [4]), we feel that it is useful to explain how the algebraic method applies to $\left(\boldsymbol{Z}^{2}, P_{\alpha}\right)$.

Given a positive, periodic irrational number $\alpha$, we can view $\left(\boldsymbol{Z}^{2}, P_{\alpha}\right)$ as $G=\{m+n \alpha: m, n \in \boldsymbol{Z})$, a dense subgroup of $\boldsymbol{R}$ with the usual ordering. Since $1 \in G^{+}$, an order automorphism of $G$ must be the form

$$
g \in G \longmapsto u g
$$

where $u, u^{-1} \in G^{+}$are algebraic units, and conversely any such $u$ determines an automorphism of $G$. It is important to know the connection between the algebraic units in $G^{+}$and the result of Theorem 2.3. For this, let

$$
\alpha=\left[\dot{a}_{0}, \cdots, \dot{a}_{L-1}, \cdots\right]
$$

be the simple continued fraction of $\alpha$. Define $p_{n}, q_{n}$ as before. We know from $\S 2$ and Theorem 1.4 that

$$
q_{k L-1} \alpha^{2}+\left(q_{k L-2}-p_{k L-1}\right) \alpha-p_{k L-2}=0 .
$$

Thus we have (recall that $\left.p_{n} q_{n-1}-p_{n-1} q_{n}=(-1)^{n-1}\right)$.

$$
\begin{aligned}
& q_{k L-1}^{2} \alpha^{2}+q_{k L-1}\left(q_{k L-2}-p_{k L-1}\right) \alpha-p_{k L-2} q_{k L-1}=0 \\
\Longrightarrow & p_{k L-1} q_{k L-2}-p_{k L-2} q_{k L-1}=p_{k L-1} q_{k L-2}+q_{k L-1}\left(p_{k L-1}-q_{k L-2}\right) \alpha \\
& -q_{k L-1}^{2} \alpha^{2} \\
\Longrightarrow & (-1)^{k L}=\left(p_{k L-1}-q_{k L-1} \alpha\right)\left(q_{k L-2}+q_{k L-1} \alpha\right) \\
\Longrightarrow & 1=\left[(-1)^{k L}\left(p_{k L-1}-q_{k L-1} \alpha\right)\right]\left(q_{k L-2}+q_{k L-1} \alpha\right) .
\end{aligned}
$$

Hence $(-1)^{k L}\left(p_{k L-1}-q_{k L-1} \alpha\right)$ is an algebraic unit with $q_{k L-2}+q_{k L-1} \alpha$ 
as its inverse. Using Theorem 2.3 and the above observation, we have the following.

THEOREM 3.1. The positive algebraic units in $G$ form a group $\mathscr{Z}_{G}$, which is generated by $(-1)^{L}\left(p_{L-1}-q_{L-1} \alpha\right)$. Moreover, we have

(1) $\left[(-1)^{L}\left(p_{L-1}-q_{L-1} \alpha\right)\right]^{k}=(-1)^{k L}\left(p_{k L-1}-q_{k L-1} \alpha\right)$,

(2) $\left[(-1)^{L}\left(p_{L-1}-q_{L-1} \alpha\right)\right]^{-k}=q_{k L-2}+q_{k L-1} \alpha$ for $k \geqq 1$.

Proof. It is clear that the positive algebraic units in $G$ form a groups. It is cyclic (for this, we use the fact that the positive units of a real quadratic field form a cyclic group [9, p. 63, Prop. 1]). Thus, it suffices to show that $(-1)^{L}\left(p_{L-1}-q_{L-1} \alpha\right)$ is the generator and the identity (1) holds.

Let us first prove (1): Assume that (1) holds for $k$,

$$
\begin{aligned}
{\left[(-1)^{L}\left(p_{L-1}-q_{L-1} \alpha\right)\right]^{k+1}=} & (-1)^{(k+1) L}\left(p_{k L-1}-q_{k L-1} \alpha\right)\left(p_{L-1}-q_{L-1} \alpha\right) \\
= & (-1)^{(k+1) L}\left[p_{L-1} p_{k L L-1}-\left(p_{L-1} q_{k L-1}+p_{k L-1} q_{L-1}\right) \alpha\right. \\
& \left.+q_{L-1} q_{k L-1} \alpha^{2}\right]
\end{aligned}
$$

where, by using the equalities in Lemma 2.2 and (3.2), we have

$$
\begin{aligned}
p_{L-1} & p_{k L-1}-\left(p_{L-1} q_{k L-1}+p_{k L-1} q_{L-1}\right) \alpha+q_{L-1} q_{k L-1} \alpha^{2} \\
& =p_{L-1} p_{k L L-1}-\left(p_{L-1} q_{k L-1}+p_{k L-1} q_{L-1}\right) \alpha+q_{L-1}\left[p_{k L-2}+\left(p_{k L-1}-q_{k L-2}\right) \alpha\right] \\
& =\left(p_{L-1} p_{k L-1}+q_{L-1} p_{k L-2}\right)-\left(p_{L-1} q_{k L-1}+q_{L-1} q_{k L-2}\right) \alpha \\
& =p_{(k+1) L-1}-q_{(k+1) L-1} \alpha .
\end{aligned}
$$

Thus (1) holds.

On behalf of $(-1)^{L}\left(p_{L-1}-q_{L-1} \alpha\right)$ generating $\mathscr{Z}_{G}$, letting $u+v \alpha$ be a generator of $u_{G}$ such that $(u+v \alpha)^{2}=q_{L-2}+q_{L-1} \alpha, i>0$. Then

$$
(u+v \alpha) \alpha=u^{\prime}+v^{\prime} \alpha
$$

for some $u^{\prime}, v^{\prime} \in Z$, where, as $u+v \alpha$ is an order automorphism of $G, \alpha \in G^{+}$, so we have $u^{\prime}+v^{\prime} \alpha \in G^{+}$. Since $u+v \alpha \in \mathrm{AUT} G$, the following equation

$$
(u+v \alpha)(m+n \alpha)=a+b \alpha
$$

is integrally solvable, i.e., there exist $m, n \in \boldsymbol{Z}$ such that (3.4) holds, where $a$ and $b$ are given integers. By using (3.3)

$$
\begin{aligned}
a+b \alpha & =(u+v \alpha)(m+n \alpha) \\
& =u m+(v m+u n) \alpha+n v \alpha^{2} \\
& =u m+(v m+u n) \alpha+n\left[u^{\prime}+v^{\prime} \alpha-u \alpha\right] \\
& =\left(u m+n u^{\prime}\right)+\left(v m+n v^{\prime}\right) \alpha
\end{aligned}
$$




$$
\Longrightarrow\left\{\begin{array}{l}
u m+u^{\prime} n=a \\
v m+v^{\prime} n=b
\end{array}\right.
$$

is solvable over $Z$ for any given $a, b \in Z$. This implies that $\operatorname{det}\left[\begin{array}{ll}u & u^{\prime} \\ v & v^{\prime}\end{array}\right]= \pm 1$. By (3.3), $\alpha=\left(v^{\prime} \alpha+u^{\prime}\right) /(v \alpha+u), v^{\prime} \alpha+u^{\prime}>0, v \alpha+$ $u>0,\left[\begin{array}{ll}v^{\prime} & v \\ u^{\prime} & u\end{array}\right] \in \operatorname{GL}(2, \boldsymbol{Z})$. Thus $\left[\begin{array}{ll}v^{\prime} & v \\ u^{\prime} & u\end{array}\right] \in \operatorname{AUT}\left(\boldsymbol{Z}^{2}, P_{\alpha}\right)$. By Theorem 2.3, $v=q_{k L-1}, u=q_{k L-2}$ for some $k \in N$. But as $(u+v \alpha)^{i}=\left(q_{k L-2}+\right.$ $\left.q_{k L-1} \alpha\right)^{i}=q_{i k L-2}+q_{i k L-1} \alpha, i k=1$, and hence $u+v \alpha=q_{L-2}+q_{L-1} \alpha$. The proof is now complete.

Finally, we wish to mention some problems: Suppose we are given a dimension group $G$ as that in Theorem 2.4, is AUT $G$ cyclic? is it generated by $\Psi$ ? If not, what can we tell about the AUT $G$ from $\Psi$ ? How to distinguish two dimension groups arised in the same manner as that in Theorem 2.4? The answers of these questions would be helpful for the classification of topological Markov chains considered by Krieger and Cuntz.

\section{REFERENCES}

1. O. Bratteli, Joductive limits of finite dimensional $C^{*}$-algebras, Trans. Amer. Math. Soc., 171 (1972).

2. E. G. Effros and J. Rosenberg, C*algebras with approximately inner fip, Pacific J. Math., 77 (1978).

3. E. G. Effros and C. L. Shen, Approximately finite $C^{*}$-algebras and continued fractions, Indiana University J. Math., 29 (1980).

4. Dimension groups and finite difference equations, J. of Operator Theory, 2 (1979).

5. E. G. Effros, D. Handelman and C. L. Shen, Dimension groups and their affine representations, Amer. J. Math., 102 (1980).

6. G. Elliott, On the classification of inductive limits of sequence of semi-simple finite dimensional algebras, J. Algebra, 38 (1976).

7. L. Fuchs, Riesz groups, Annali della Scuola Norm. Sup., Pisa, 19 (1965).

8. G. H. Hardy and E. M. Wright, An Introduction to the Theory of Numbers, 4th ed., (1975), Oxford.

9. P. Samuels, Algebraic Theory of Numbers, Hermann, 1969.

10. C. L. Shen, On the classification of the ordered groups associated with the approxi. mately finite dimensional C*-algebras, Duke Math. J., 46 (1979).

Received May 25, 1979.

SUNY

Stony Brook, NY 11794 



\section{PACIFIC JOURNAL OF MATHEMATICS}

\section{EDITORS}

DONALD BABBITT (Managing Editor)

University of Galifornia

Los Angeles, California 90024

Hugo RossI

University of Utah

Salt Lake City, UT 84112

C. C. MoOre AND ANDrew OGG

University of California

Berkeley, CA 94720

\section{J. DugundjI}

Department of Mathematics University of Southern California

Los Angeles, California 90007

R. Finn and J. Milgram

Stanford University

Stanford, California 94305

ASSOCIATE EDITORS

E. F. Beckenbachi

B. H. NeumanN

F. WOLF

K. YOSHIDA

\section{SUPPORTING INSTITUTIONS}

UNIVERSITY OF BRITISH COLUMBIA

CALIFORNIA INSTITUTE OF TECHNOLOGY

UNIVERSITY OF CALIFORNIA

MONTANA STATE UNIVERSITY

UNIVERSITY OF NEVADA, RENO

NEW MEXICO STATE UNIVERSITY

OREGON STATE UNIVERSITY

UNIVERSITY OF OREGON
UNIVERSITY OF SOUTHERN CALIFONIA

STANFORD UNIVERSITY

UNIVERSITY OF HAWAII

UNIVERSITY OF TOKYO

UNIVERSITY OF UTAH

WASHINGTON STATE UNIVERSITY

UNIVERSITY OF WASHINGTON

Printed in Japan by International Academic Printing Co., Ltd., Tokyo, Japan 


\section{Pacific Journal of Mathematics \\ Vol. 89 , No. 1 \\ May, 1980}

David Bressoud, A note on gap-frequency partitions ................ 1

John David Brillhart, A double inversion formula ................. 7

Frank Richard Deutsch, Günther Nürnberger and Ivan Singer, Weak

Chebyshev subspaces and alternation .......................

Edward Richard Fadell, The relationship between Ljusternik-Schnirelman

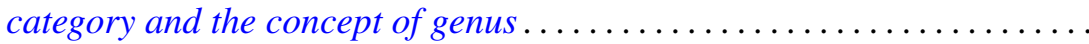

Harriet Jane Fell, On the zeros of convex combinations of polynomials.......

John Albert Fridy, An addendum to: "Tauberian theorems via block

dominated matrices" ..................................

Andrzej Granas, Ronald Bernard Guenther and John Walter Lee, Applications of topological transversality to differential equations. I. Some nonlinear

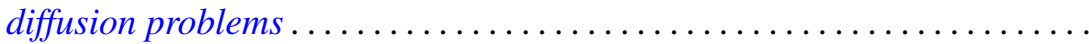

David E. Handelman and G. Renault, Actions of finite groups on self-injective

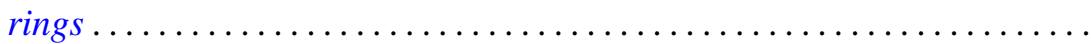

Michael Frank Hutchinson, Local $\Lambda$ sets for profinite groups .............

Arnold Samuel Kas, On the handlebody decomposition associated to a

Lefschetz fibration...

Hans Keller, On the lattice of all closed subspaces of a Hermitian space.....

P. S. Kenderov, Dense strong continuity of pointwise continuous

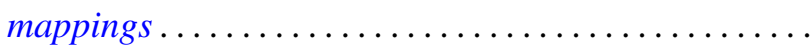

Robert Edward Kennedy, Krull rings.................

Jean Ann Larson, Richard Joseph Laver and George Frank McNulty,

Square-free and cube-free colorings of the ordinals ...

Viktor Losert and Harald Rindler, Cyclic vectors for $L^{p}(G)$

John Rowlay Martin and Edward D. Tymchatyn, Fixed point sets of

1-dimensional Peano continua...

Augusto Nobile, On equisingular families of isolated singularities ...

Kenneth Joseph Prevot, Imbedding smooth involutions in trivial bundles ...

Thomas Munro Price, Spanning surfaces for projective planes in four space.

Dave Riffelmacher, Sweedler's two-cocycles and Hochschild cohomology....

Niels Schwartz, Archimedean lattice-ordered fields that are algebraic over

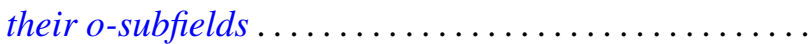

Chao-Liang Shen, A note on the automorphism groups of simple dimension groups.

Kenneth Barry Stolarsky, Mapping properties, growth, and uniqueness of

Vieta (infinite cosine) products ...

Warren James Wong, Maps on simple algebras preserving zero products. I.

The associative case ............................. 